\title{
Research Paper: The Efficacy of Platelet-Rich Plasma in the Treatment of Hemophilic Arthropathy
}

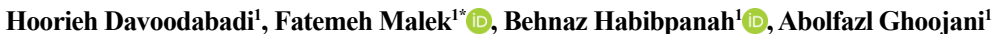

1. Pediatric Congenital Hematologic Disorders Research Center, Research Institute for Childern's Health, Shahid Beheshti University of Medical Sciences, Tehran.

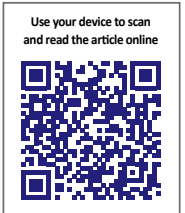

Citation Davoodabadi H, Malek F, Habibpanah B, Ghoojani A. The Efficacy of Platelet-Rich Plasma in the Treatment of Hemophilic Arthropathy. Journal of Research in Orthopedic Science. 2020; 7(4):165-168. http://dx.doi.org/10.32598/JROSJ.7.4.693.1

http://dx.doi.org/10.32598/JROSJ.7.4.693.1

Keywords:

Arthropathies, PlateletRich plasma, Prophylaxis, Synovitis, Hemophilia

\begin{abstract}
A B S T RA C T
Background: The most clinical manifestation of hemophilia, as a congenital bleeding disorder, is the Intra-articular hemorrhage. The treatment of acute hemarthrosis with a sufficient amount of factor replacement will return the joint to the normal status.

Objectives: This study aimed to evaluate the efficacy of platelet-rich plasma in the treatment of hemophilic arthropathy patients to reduce and improve their pain and physical function.

Methods: Consecutive patients with hemophilic arthropathy were included in a self-control openlabel trial in Mofid Children's Hospital, Tehran, Iran. All participants received three platelet-rich plasma injections given two weeks apart. Also, the seriate self-reported and objective clinical outcomes were evaluated before and three months after the treatment.

Results: A total of 11 patients with hemophilic arthropathy involving 14 joints received three injections of platelet-rich plasma at 2-week intervals, at our center. All patients were evaluated with a visual analog scale, the hemophilia joint health score, and the range of motion, and bleeding events, before and after the treatment. Finally, 9 boys $(81.8 \%)$ and 2 girls $(18.2 \%)$ completed the study. The mean age of the participants was 14.7 years (range, 9-19 years). In all patients, statistically significant improvements were noted on all evaluated measures.

Conclusion: Platelet-rich plasma therapy is a reasonable and minimally invasive procedure that includes beneficial chondro-regenerative properties.
\end{abstract}

\section{Introduction}

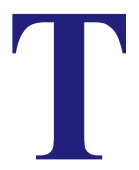

he most clinical manifestation of hemophilia, as a congenital bleeding disorder, is the Intra-articular hemorrhage [1]. The treatment of acute hemarthrosis with a sufficient amount of factor replacement will return the joint to the normal status [2].
However, frequent bleeding events in a specific joint cause the target joint formation and consequently chronic synovitis that is defined as a triangle consisted of synovial inflammation, recurrent hemarthrosis, and synovial hypertrophy $[2,3]$. To make things worse, the mentioned vicious cycle does not repair itself. Hence, chronic synovitis without any specified treatment inevitably destroys the articular cartilage and leads to a progressive arthropathy [2-4].

* Corresponding Author:

Fatemeh Malek, MD.

Address: Pediatric Congenital Hematologic Disorders Research Center, Research Institute for Childern's Health, Shahid Beheshti University of Medical Sciences, Tehran.

E-mail:fmalek7721@gmail.com 
The surgical synovectomies and radiosynovectomy have limited effects on the destructed cartilage of affected joints. Hence, invasive surgical methods, such as arthroplasty appear to be the only therapeutic option [3-7]. However, Bayliss et al. highlighted the increasing failure rate of implants at younger ages. Consequently, the number of revisions increases, and unfortunately, these patients may become a burden to society because of the disability and cost [3]. Therefore, we hypothesize that the administration of regenerative methods heralds a new era for the treatment of young patients with hemophilic arthropathy.

Platelet-Rich Plasma (PRP) is an autologous concentration of platelets in a limited volume of plasma and is prepared by centrifugation of blood. Platelets contain a tremendous amount of growth factors and cytokines, which have the potential of cellular growth stimulation, vascularization, proliferation, tissue regeneration, and collagen synthesis. The PRP distributes high concentrations of cytokines and growth factors into the damaged tissues; this distribution beneficially affects the cartilage tissue regeneration [8]. Therefore, we investigated the efficacy of PRP administration in the control of the symptoms and the disease activity of hemophilic arthropathy.

\section{Materials and Methods}

This open-label self-controlled trial was conducted in Mofid Children's Hospital, Tehran, Iran. The inclusion criteria were the proven diagnosis of hemophilic arthropathy with imaging and physical examination and the history of synoviorthesis or synovectomy. Patients who met the inclusion criteria were invited to participate.

The patients and their affected joints were evaluated with a visual analog score, the Hemophilia Joint Health Score (HJHS), and the range of motion and bleeding events, before and three months after the treatment. Also, the evaluation of the patients was performed by the same team for the whole time frame. The obtained data were analyzed with the D'Agostino-Pearson test for normality and paired t-tests using GraphPad Prism software.

\section{PRP preparation}

Before the PRP injection, the patients received their required factor to bring the factor level up to $80 \%$. Additionally, patients with inhibitors received bypassing agents. Next, the extracted blood (from the patients or their donors) was centrifuged at $1200 \mathrm{rpm}$, for $15 \mathrm{~min}$ utes. After the appearance of the platelet cloud, the blood was centrifuged at $2600 \mathrm{rpm}$, for 30 minutes. Then, the injection was initiated and the process repeated three times with 2-week intervals. Also, the joints were evaluated one week after the last injection and three months later.

\section{Results}

Eleven patients, including 9 boys (81.8\%) and 2 girls (18.9\%) with 14 involved joints were treated at our center, between December 2015 and December 2017. The mean age of the patients was 14.7 years (range, 9-19 years). Also, six, one, and five patients were affected by hemophilia A, hemophilia B, and Von Willebrand type 3 disease, respectively. Three patients $(27 \%)$ had inhibitors. Out of 14 joints included in the study, 10 were ankles $(71.4 \%)$, and four were knees $(28.6 \%)$. Furthermore, three patients had two affected joints.

The average HJHS score was $7.357 \pm 1.33$ and $2.714 \pm 1.13$ before and three months after the injection, respectively. In this regard, the paired t-test revealed a statistically significant difference $(\mathrm{P}=0.0001)$. Although two patients $(18.18 \%)$ had bleeding events after the PRP injection, all the patients reported a considerable pain reduction, based on the visual analog score $(\mathrm{P}=0.0005)$. Moreover, the range of motion (Mean $\pm \mathrm{SEM}, \mathrm{n})$ significantly differ before $(46.85 \pm 16.81,13)$ and $\operatorname{after}(51.85 \pm 17.04,13)$ the PRP $(\mathrm{P}<0.0088)$. Besides, no significant complication was observed during the treatment and follow-up.

This study aimed to evaluate the effectiveness of PRP treatment on the reduction of pain and the improvement of physical function in hemophilic arthropathy patients. During the treatment and the follow-up, the HJHS, and the range of motion of the patients improved, compared with the preinjection values. This study revealed that PRP is a useful and safe treatment option to control the activity of hemophilic arthropathy [9].

Teissler et al. reported the PRP treatment results in 6 patients with synovitis in 8 joints [10]. The present study differs from the study of Teissler et al. in various aspects, such as the number of patients. The mentioned study included a fewer number of patients, the affected joints of whom were limited to ankle. Furthermore, the HJHS score was not significantly changed after the PRP treatment [10]. Caviglia et al. studied 19 patients with 28 affected joints on different parts of the body.

These authors reported statistically significant decreases $(\mathrm{P}<0.001)$ in the HJHS score, joint bleeding episodes, pain relief (visual analog score), and joint perimeter $[11,12]$. Although the above-mentioned study is more comprehensive than ours, the present study included the patients with Von Willebrand and a lower mean of age, 
who belonged to the pediatric age group. We concluded that PRP is a safe inexpensive treatment that includes beneficial chondro-regenerative properties.

\section{Ethical Considerations}

\section{Compliance with ethical guidelines}

This study was conducted based in the ethical guidelines of the Declaration of Helsinki, 1975 (Code: IR.SBMU. RETECH.REC.1396.326). An informed written consent was obtained. The participants were informed about the purpose of the research and its implementation stages; they were also assured about the confidentiality of their information; Moreover, They were allowed to leave the study whenever they wish, and if desired, the results of the research would be available to them.

\section{Funding}

This research did not receive any specific grant from funding agencies in the public, commercial, or not-forprofit sectors.

\section{Authors' contributions}

All authors contributed in preparing this research.

\section{Conflict of interest}

The authors declared no conflict of interest.

\section{Acknowledgments}

The authors would like to thank to Dr. Elham Sadeghi, for her contribution in data gathering.

\section{References}

[1] Eshghi P, Abolghasemi H, Malek F, Naderi M, Panahi Y, Habibpanah B, et al. A prospective crossover triple-blind controlled trial on the safety and efficacy of Iranian recombinant FVIII (Safacto) versus plasma derived FVIII; A pilot study. Iran J Blood Cancer. 2015; 7(4): 171-4. http://ijbc.ir/article-1-624-en.html

[2] Melchiorre D, Manetti M, Matucci-Cerinic M. Pathophysiology of hemophilic arthropathy. J Clin Med. 2017; 6(7):63. [DOI:10.3390/jcm6070063] [PMID] [PMCID]

[3] Bayliss LE, Culliford D, Monk AP, Glyn-Jones S, Prieto-Alhambra D, Judge A, et al. The effect of patient age at intervention on risk of implant revision after total replacement of the hip or knee: A population-based cohort study. Lancet. 2017; 389(10077):1424-30. [DOI:10.1016/S0140-6736(17)300594] [PMID] [PMICD]
[4] Rodriguez-Merchan EC, Valentino LA. Orthopedic disorders of the knee in hemophilia: A current concept review. World J Orthop. 2016; 7(6):370-5. [DOI:10.5312/wjo.v7.i6.370] [PMID] [PMCID]

[5] Solimeno LP, Pasta G. Knee, and Ankle Arthroplasty in Hemophilia. J Clin Med. 2017; 6(11):107. [DOI:10.3390/ jcm6110107] [PMID] [PMCID]

[6] Eshghi P, Goudarzipour K, Davoudabadi FH, Aghakhani $R$. Intra articular injection of rifampin in Iranian children with Haemophilic arthropathy. Iran J Blood Cancer. 2010 2(3):123-7. http://ijbc.ir/article-1-242-en.html

[7] Özcan Z. Radiosynovectomy in hemophilic synovitis. Mol Imaging Radionucl Ther. 2014; 23(1):1-4. [DOI:10.4274/ Mirt.49369] [PMID] [PMCID]

[8] Glynn LG, Mustafa A, Casey M, Krawczyk J, Blom J, Galvin $\mathrm{R}$, et al. Platelet-Rich Plasma (PRP) therapy for knee arthritis: A feasibility study in primary care. Pilot Feasibility Stud. 2018; 4:93 [DOI:10.1186/s40814-018-0288-2] [PMID] [PMCID]

[9] Kavadarci G, Demircioglu DT, Celik MY, Emre TY. Effectiveness of platelet-rich plasma in the treatment of moderate knee osteoarthritis: A randomized prospective study. J Phys Ther Sci. 2015; 27(12):3863-7. [DOI:10.1589/jpts.27.3863] [PMID] [PMCID]

[10] Teyssler P, Kolostova K, Bobek V. The impact of plateletrich plasma on chronic synovitis in hemophilia. Acta Orthop Belg. 2014; 80(1):11-7. [PMID]

[11] Caviglia H, Landro ME, Daffunchio C, Galatro G, Douglas Price AL, Salgado P, et al. Platelet rich plasma for chronic synovitis treatment in patients with haemophilia. Haemophilia. 2017; 23(4):613-9. [DOI:10.1111/hae.13212] [PMID]

[12] Li TY. Platelet-rich plasma intra-articular injection in treating hemophilic arthropathy. Bethesda: Clinical Trials; 2017. https://clinicaltrials.gov/ct2/show/NCT02601170 
This Page Intentionally Left Blank 